Research Article

\title{
Time-Fractional Klein-Gordon Equation with Solitary/Shock Waves Solutions
}

\author{
Sayed Saifullah $\mathbb{D}^{1},{ }^{1}$ Amir Ali $\mathbb{D},{ }^{1}$ Muhammad Irfan $\mathbb{D}^{2},{ }^{2}$ and Kamal Shah $\mathbb{D}^{1}$ \\ ${ }^{1}$ Department of Mathematics, University of Malakand, Chakdara Dir (L), Khyber Pakhtunkhwa, Malakand, Pakistan \\ ${ }^{2}$ Department of Physics, University of Malakand, Chakdara Dir (L), Khyber Pakhtunkhwa, Malakand, Pakistan \\ Correspondence should be addressed to Kamal Shah; kamalshah408@gmail.com
}

Received 1 June 2021; Accepted 22 September 2021; Published 13 October 2021

Academic Editor: Libor Pekař

Copyright $\odot 2021$ Sayed Saifullah et al. This is an open access article distributed under the Creative Commons Attribution License, which permits unrestricted use, distribution, and reproduction in any medium, provided the original work is properly cited.

In this article, we study the time-fractional nonlinear Klein-Gordon equation in Caputo-Fabrizio's sense and Atangana-Baleanu-Caputo's sense. The modified double Laplace transform decomposition method is used to attain solutions in the form of series of the proposed model under aforesaid fractional operators. The suggested method is the composition of the double Laplace transform and decomposition method. The convergence of the considered method is demonstrated for the considered model. It is observed that the obtained solutions converge to the exact solution of the proposed model. For validity, we consider two particular examples with appropriate initial conditions and derived the series solution in the sense of both operators for the considered model. From numerical solutions, it is observed that the considered model admits pulse-shaped solitons. It is also observed that the wave amplitude enhances with variations in time, which infers the coefficient $\alpha$ significantly increases the wave amplitude and affects the nonlinearity/dispersion effects, therefore may admit monotonic shocks. The physical behavior of the considered numerical examples is illustrated explicitly which reveals the evolution of localized shock excitations.

\section{Introduction}

In recent years, the fractional-order calculus is extensively used as a promising tool in numerous areas of physical sciences [1-3] due to its extensive applications to study a diversity of real world phenomena in bioengineering [4-6], electronics [7, 8], visco-elasticity [9], robotic technology [10], signal processing [11], control theory [12], diffusion model, and relaxation processes [13-15]. In fractional-order calculus, the order of derivatives and integrals is arbitrary [16]. Therefore, the fractional-order nonlinear partial differential equations (FNPDEs) have established a fundamental interest to generalize an integer-order nonlinear partial differential equations (NPDEs) to represent complex problems in engineering, thermodynamics, optical physics, and fluid dynamics $[17,18]$.

The most significant advantage of using fractional differential equations is their nonlocal property with memory preserving [2, 19]. It is assumed that the integer-order differential operators are local but fractional are nonlocal because fractional differential operators are global as they converge to ordinary differential operator when fractionalorder becomes one [20]. This implies that not only the following condition of the system depends on its present condition but also on all its past states appropriate to memory and hereditary property [21].

The nonlinear Klein-Gordon equation considered herein was first proposed to describe relativistic electrons by the well-known physicists O. Klein and W. Gordon in 1926, while Klein-Gordon model was originally studied for quantum waves by Schrödinger [15, 22]. The Klein-Gordon equation has many applications in quantum mechanics, quantum field theory, relativistic physics, solid-state physics, plasma physics, nonlinear optics, dispersive wave-phenomena, and condensed type matter physics and also has soliton type solution [23-28].

Here, we consider the TFKG equation [29, 30]: 


$$
\frac{\partial^{\alpha} \phi(x, t)}{\partial t^{\alpha}}-\frac{\partial^{2} \phi(x, t)}{\partial x^{2}}+c \phi+b g(\phi(x, t))=h(x, t), \quad 1<\alpha \leq 2,
$$

with initial conditions

$$
\begin{aligned}
\phi(x, 0) & =f(x), \\
\phi_{t}(x, t) & =g(x),
\end{aligned}
$$

where $\phi$ is a function of spatial and temporal variables $x$ and $t$. The function $g(\phi(x, t))$ contains the nonlinearity present in the model, $b$ and $c$ are real numbers, and $h$ is an analytic/ source function.

There are several fractional operators that have been studied in fractional calculus. For example, Caputo, Caputo-Fabrizio, and Atangana-Baleanu-Caputo's sense $[31,32]$. These operators are very useful because of the complexity of fractional nonlinear differential equations (FNDEs), where classical operators cannot solve such equations to obtain explicit solutions. Due to this disadvantage of the classical operators, one needs a legitimate numerical method to obtain the coefficients of the series solutions of FNDEs [33-35]. The Caputo fractional operator is used widely in applied sciences, but this operator has some disadvantages about the singularity. To overcome this problem, Caputo and Fabrizio introduced a nonsingular fractional operator, using an exponential decay kernel. Similarly, another form of nonsingular and nonlocal fractional operator known as Atangana-Baleanu fractional operator was introduced, which produces efficient results due to the nonlocal and nonsingular kernel [36].

There are numerous methods that have been used to find numerical as well as analytical solutions to FNDEs, for example, Laplace transforms method [37], Adomian decomposition method (ADM) [38, 39], multistep approach [40], double Laplace transform [41, 42], integral transform [43], iterative reproducing kernel method [44], homotopy perturbation method (HPM) [45, 46], Sumudu transform method (STM) [47], and variational iteration method (VIM) $[48,49]$.

Similarly, the integer-order Klein-Gordon equation has been widely investigated by applying the inverse scattering method, variation iteration method [50, 51], Bäcklund transformation method, modified decomposition method and modified Adomain decomposition method [52], auxiliary equation method [53], homotopy perturbation transform method [54], radial basis function [55], homotopy analysis technique [56], sine-cosine and tanh-sech methods [57], pseudospectral method [58], and Hirota bilinear forms [59] together with the Jacobian elliptical function [60]. We will use a modified double Laplace transform to find the approximate solutions of the proposed model in CF and ABC sense. It should be noted that numerous numerical and analytical approaches have also been applied to study TFKG equations with Caputo and Riemann-Liouville (RL) operators $[61,62]$. The advantage of the proposed technique is that it converges to an exact solution of a problem after some iterations and does not involve any perturbation or discretization.
The remainder of this article is organized as follows: In Section 2, some basic definitions are given associated with the fractional calculus. In Section 3, we present the solution to the nonlinear Klein-Gordon equation in CF and ABC by using the proposed method (MDLDM). In Section 4, convergence of the proposed method for the considered model is discussed. In Section 5, we present two numerical examples related to equation (1). In Section 6, we accomplish the article.

\section{Preliminaries}

Here, we provide some basic definitions, which will be used throughout the article.

Definition 1. Let $\phi \in H^{1}(a, b)$ and $\alpha \in(0,1]$, then the Caputo-Fabrizio operator of fractional order can be written as [31]

$$
{ }^{\mathrm{CF}} D^{\alpha} \phi(t)=\frac{M(\alpha)}{1-\alpha} \int_{a}^{t} \phi^{\prime}(t) \exp \left(\frac{-\alpha(t-s)}{1-s}\right) \mathrm{d} s,
$$

such that $M(0)=M(1)=1$. It should be noted that $M(\alpha)$ is the normalized function. When $\phi(t) \notin H^{1}(a, b)$, then the above definition can be formulated for $\phi \epsilon L^{-1}(-\infty, b)$ for any $\alpha \epsilon[0,1]$ as

$$
{ }^{\mathrm{CF}} D^{\alpha} \phi(t)=\frac{\alpha M(\alpha)}{1-\alpha} \int_{-\infty}^{t}\left(\phi^{\prime}(t)-\phi(s)\right)-\exp \left(\frac{-\alpha(t-s)}{1-s}\right) \mathrm{d} s .
$$

Definition 2. Let $\phi \in H^{1}(a, b)$, with $b>a$ and $\alpha \in[0,1]$, then the fractional-order operator in Atangana-Baleanu-Caputo's sense is defined as [32]

$$
{ }^{\mathrm{ABC}} D^{\alpha} \phi(t)=\frac{B(\alpha)}{(1-\alpha)} \int_{a}^{t} \phi^{\prime}(s) E_{\alpha}\left(\frac{-\alpha(t-s)^{\alpha}}{(1-\alpha)}\right) \mathrm{d} s,
$$

where ${ }^{\mathrm{ABC}} D^{\alpha} \phi(t)$ is a fractional operator with Mittag-Leffler kernel in Caputo's sense. $B(\alpha)$ is called normalization function having properties $B(0)=B(1)=1$.

Remark 1. For Definitions 1 and 2, $n=[\alpha]+1,[\alpha]$ is the greatest integer not greater than $\alpha$ and $\Gamma$ is the well-known gamma function which is defined as

$$
\Gamma(\alpha)=\int_{0}^{\infty} e^{-s} s^{\alpha-1} \mathrm{~d} s
$$

Definition 3. Consider a function $\phi(x, t)$ for $x, t>0$ in $x, t$ plane; the double Laplace transform of the function $\phi(x, t)$ as given by [63] is defined by

$$
\mathscr{L}_{x} \mathscr{L}_{t}[\phi(x, t)]=\int_{0}^{\infty} e^{-p x} \int_{0}^{\infty} e^{-s t} \phi(x, t) \mathrm{d} t \mathrm{~d} x,
$$

where $p$ and $s$ are the complex numbers.

Definition 4. Application of the double Laplace on fractional-order operator in Caputo-Fabrizio sense is given by 


$$
\begin{array}{r}
\mathscr{L}_{x} \mathscr{L}_{t}\left\{{ }^{\mathrm{CF}} D_{x}^{\alpha+n} \phi(x, t)\right\}=\frac{M(\alpha)}{p+(1-p) \alpha}\left[p^{n+1} \bar{\phi}(p, s)-\sum_{k=0}^{n} p^{n-k} \mathscr{L}_{t}\left\{\frac{\partial^{k} \phi(0, t)}{\partial x^{k}}\right\}\right], \\
\mathscr{L}_{x} \mathscr{L}_{t}\left\{{ }^{\mathrm{CF}} D_{t}^{\beta+m} \phi(x, t)\right\}=\frac{M(\beta)}{s+(1-s) \beta}\left[s^{m+1} \bar{\phi}(p, s)-\sum_{k=0}^{m} s^{m-k} \mathscr{L}_{x}\left\{\frac{\partial^{k} \phi(x, 0)}{\partial t^{k}}\right\}\right],
\end{array}
$$

where $m \& n=0,1,2, \ldots$ and $\bar{\phi}(p, s)=\mathscr{L}_{x} \mathscr{L}_{t}\{\phi(x, t)\}$.

Definition 5. Application of double Laplace transform on fractional-order operator in Atangana-Baleanu-Caputo's sense is given by

$$
\begin{aligned}
& \mathscr{L}_{x} \mathscr{L}_{t}\left\{{ }^{\mathrm{ABC}} D_{x}^{\alpha} \phi(x, t)\right\}=\frac{B(\alpha)}{(1-\alpha)\left(p^{\alpha}+(\alpha /(1-\alpha))\right)}\left[p^{\alpha} \bar{\phi}(p, s)-\sum_{k=0}^{n-1} p^{\alpha-1-k} \mathscr{L}_{t}\left\{\frac{\partial^{k} \phi(0, t)}{\partial x^{k}}\right\}\right] \\
& \mathscr{L}_{x} \mathscr{L}_{t}\left\{{ }^{\mathrm{ABC}} D_{t}^{\beta} \phi(x, t)\right\}=\frac{B(\beta)}{(1-\beta)\left(s^{\beta}+(\beta /(1-\beta))\right)}\left[s^{\beta} \bar{\phi}(p, s)-\sum_{k=0}^{m-1} s^{\beta-1-k} \mathscr{L}_{x}\left\{\frac{\partial^{k} \phi(x, 0)}{\partial t^{k}}\right\}\right]
\end{aligned}
$$

where $n=[\alpha]+1, m=[\beta]+1$.

From the above definitions, we conclude that

$$
\mathscr{L}_{x} \mathscr{L}_{t} \phi(x) \phi(t)=\bar{u}(p) \bar{\phi}(s)=\mathscr{L}_{x} \phi(x) \mathscr{L}_{t} \phi(t) .
$$

The inverse double Laplace transform $\mathscr{L}_{x}^{-1} \mathscr{L}_{t}^{-1}\{\bar{\phi}(x, t)\}=\phi(x, t)$ is represented by a complex double integral formula

$$
\mathscr{L}_{x}^{-1} \mathscr{L}_{t}^{-1}\{\bar{\phi}(x, t)\}=\frac{1}{2 \pi i} \int_{c-i \infty}^{c+i \infty} e^{s t} \int_{d-i \infty}^{d+i \infty} e^{p x} \bar{\phi}(p, s) \mathrm{d} p \mathrm{~d} s,
$$

where $\bar{\phi}(p, s)$ is an analytic function $\forall p$ and $s$ is defined in the region by the inequalities $\operatorname{Re}(p) \geq c$ and $\operatorname{Re}(s) \geq d$, where $c, d \in \mathbb{R}$ is to be considered accordingly.

\section{Modified Double Laplace Transform Decomposition Method}

In this section, we briefly present the proposed method MDLDM. It is the composition of double Laplace and the Adomian decomposition method used to obtain the solution in the series form of nonlinear partial differential equations (NPDEs) and nonlinear ordinary differential equations (NODEs). It is the most effective scheme to find the approximate solution of dynamic problems. Here, first, we briefly discuss the proposed approach and then apply to equation (1). Let us suppose the general nonlinear problem of the form

$$
L \phi+R \phi+N \phi=h(x, t), \quad \forall t \in \mathbb{R},
$$

where $\phi=\phi(x, t)$ in the above system, $L$ is a linear operator, $R$ is an operator containing the linear terms, $N$ is a nonlinear operator, and $h(x, t)$ is an external function.

3.1. The Proposed Model with Exponential Decay Kernel. In this subsection, we consider equation (1) in CF sense and use the proposed method to obtain series solution to equation (1), by using the technique defined in Section 3 .

$$
{ }^{\mathrm{CF}} D_{t}^{\alpha+m} \phi-\frac{\partial^{2} \phi}{\partial x^{2}}+c \phi+b g(\phi)=h(x, t), \quad 0<\alpha \leq 1, m=1,
$$

together with the subsidiary conditions

$$
\begin{aligned}
\phi(x, 0) & =f(x), \\
\phi_{t}(x, 0) & =g(x) .
\end{aligned}
$$

Comparing equations (13) with (12), we observe that $L=\partial^{2} / \partial x^{2}, N=b g(\phi)$ contain nonlinear term, and ${ }^{\mathrm{CF}} D_{t}^{\alpha+m}$ is the fractional-order operator in Caputo-Fabrizio's sense. Applying the double Laplace transform and using the definitions given in Section 2, we obtain

$$
\mathscr{L}_{x} \mathscr{L}_{t}\left\{{ }^{\mathrm{CF}} D_{t}^{\alpha}+m \phi\right\}-\mathscr{L}_{x} \mathscr{L}_{t}\left\{\frac{\partial^{2}}{\partial x^{2}} \phi\right\}+c \mathscr{L}_{x} \mathscr{L}_{t}\{\phi\}+b \mathscr{L}_{x} \mathscr{L}_{t}\{g \phi\}=\mathscr{L}_{x} \mathscr{L}_{t} h(x, t)
$$

Using double Laplace on the fractional-order operator in Caputo-Fabrizio's sense, we obtain 


$$
\begin{aligned}
\mathscr{L}_{x} \mathscr{L}_{t}\{\phi(x, t)\}= & \left(\frac{1}{s}\right) \mathscr{L}_{x}\{\phi(x, 0)\}+\left(\frac{1}{s^{2}}\right) \mathscr{L}_{x}\left\{\phi_{t}(x, 0)\right\}+\left(\frac{s+(1-\alpha) s}{s^{2}}\right) \mathscr{L}_{x} \mathscr{L}_{t}\left\{\frac{\partial^{2}}{\partial x^{2}} \phi(x, t)\right\} \\
& +c\left(\frac{s+(1-\alpha) s}{s^{2}}\right) \mathscr{L}_{x} \mathscr{L}_{t}\{\phi(x, t)\}+b\left(\frac{s+(1-\alpha) s}{s^{2}}\right) \mathscr{L}_{x} \mathscr{L}_{t}\{g \phi(x, t)\} \\
& +\left(\frac{s+(1-\alpha) s}{s^{2}}\right) \mathscr{L}_{x} \mathscr{L}_{t} h(x, t) .
\end{aligned}
$$

Now, applying the single Laplace transform on initial conditions given in equation (14), we obtain

$$
\begin{aligned}
\mathscr{L}_{x}\{\phi(x, 0)\} & =\bar{f}(p), \\
\mathscr{L}_{x}\left\{\phi_{t}(x, 0)\right\} & =t \bar{g}(p) .
\end{aligned}
$$

Consider the series solution of the form

$$
\phi(x, t)=\sum_{n=0}^{\infty} \phi_{n}(x, t)
$$

and the nonlinear terms are decomposed as

$$
g(\phi(x, t))=\sum_{i=0}^{\infty} A_{n}
$$

where $A_{n}$ is a well-known polynomial called Adomian polynomial [64] of the functions $\phi_{0}, \phi_{1}, \phi_{2}, \ldots$, described by the formula

$$
A_{n}=\frac{1}{n !} \frac{\mathrm{d}^{n}}{\mathrm{~d} \lambda^{n}}\left[\sum_{k=0}^{n} \lambda^{k} \phi_{k}(x, t)\right]_{\lambda=0}
$$

Solving equation (12) with the help of equations (16) and (20), we obtain the following series solution:

$$
\begin{aligned}
\phi_{0}= & \mathscr{L}_{x}^{-1} \mathscr{L}_{t}^{-1}\left[\frac{1}{s} \bar{\phi}(p, 0)\right]+t \mathscr{L}_{x}^{-1} \mathscr{L}_{t}^{-1}\left[\frac{1}{s^{2}} \bar{\phi}(p, 0)\right]=\phi(x, 0), \\
\phi_{1}= & \mathscr{L}_{x}^{-1} \mathscr{L}_{t}^{-1}\left[\left(\frac{s+(1-s) \alpha}{s^{2}}\right) \mathscr{L}_{x} \mathscr{L}_{t}\left\{\phi_{0 x x}\right\}\right]-c \mathscr{L}_{x}^{-1} \mathscr{L}_{t}^{-1}\left[\left(\frac{s+(1-s) \alpha}{s^{2}}\right) \mathscr{L}_{x} \mathscr{L}_{t}\left\{\phi_{0}\right\}\right] \\
& -b \mathscr{L}_{x}^{-1} \mathscr{L}_{t}^{-1}\left[\left(\frac{s+(1-s) \alpha}{s^{2}}\right) \mathscr{L}_{x} \mathscr{L}_{t}\left\{A_{0}\right\}\right]+\mathscr{L}_{x}^{-1} \mathscr{L}_{t}^{-1}\left[\left(\frac{s+(1-s) \alpha}{s^{2}}\right) \mathscr{L}_{x} \mathscr{L}_{t}\{h(x, t)\}\right], \\
\phi_{2}= & \mathscr{L}_{x}^{-1} \mathscr{L}_{t}^{-1}\left[\left(\frac{s+(1-s) \alpha}{s^{2}}\right) \mathscr{L}_{x} \mathscr{L}_{t}\left\{\phi_{1 x x}\right\}\right]-c \mathscr{L}_{x}^{-1} \mathscr{L}_{t}^{-1}\left[\left(\frac{s+(1-s) \alpha}{s^{2}}\right) \mathscr{L}_{x} \mathscr{L}_{t}\left\{\phi_{1}\right\}\right] \\
& -b \mathscr{L}_{x}^{-1} \mathscr{L}_{t}^{-1}\left[\left(\frac{s+(1-s) \alpha}{s^{2}}\right) \mathscr{L}_{x} \mathscr{L}_{t}\left\{A_{1}\right\}\right], \\
\phi_{3}= & \mathscr{L}_{x}^{-1} \mathscr{L}_{t}^{-1}\left[\left(\frac{s+(1-s) \alpha}{s^{2}}\right) \mathscr{L}_{x} \mathscr{L}_{t}\left\{\phi_{2 x x}\right\}\right]-c \mathscr{L}_{x}^{-1} \mathscr{L}_{t}^{-1}\left[\left(\frac{s+(1-s) \alpha}{s^{2}}\right) \mathscr{L}_{x} \mathscr{L}_{t}\left\{\phi_{2}\right\}\right] \\
& -b \mathscr{L}_{x}^{-1} \mathscr{L}_{t}^{-1}\left[\left(\frac{s+(1-s) \alpha}{s^{2}}\right) \mathscr{L}_{x} \mathscr{L}_{t}\left\{A_{2}\right\}\right] .
\end{aligned}
$$

The other terms can be calculated in a similar way. The final solution can be written as

$$
\phi(x, t)=\sum_{n=0}^{\infty} \phi_{n}(x, t) .
$$

3.2. The Proposed Model with Mittag-Leffler Kernel. Here, we consider equation (1) in ABC sense and applying the proposed method with definitions discussed in Section 2,

$$
{ }^{\mathrm{ABC}} D_{t}^{\alpha} \phi(x, t)-\frac{\partial^{2} \phi}{\partial x^{2}}+c \phi+b g(\phi)=h(x, t), \quad \forall t \in \mathbb{R}, 1<\alpha \leq 2,
$$

with subsidiary conditions

$$
\begin{aligned}
\phi(x, 0) & =f(x), \\
\phi_{t}(x, 0) & =g(x) .
\end{aligned}
$$

Solving equation (23) with the techniques used in Section 3, we obtain the following series solution: 


$$
\begin{aligned}
\phi_{0}= & \mathscr{L}_{x}^{-1} \mathscr{L}_{t}^{-1}\left[\frac{1}{s} \bar{\phi}(p, 0)\right]+t \mathscr{L}_{x}^{-1} \mathscr{L}_{t}^{-1}\left[\frac{1}{s^{2}} \bar{\phi}(p, 0)\right]=\phi(x, 0), \\
\phi_{1}= & \mathscr{L}_{x}^{-1} \mathscr{L}_{t}^{-1}\left[\left(1-\alpha+\frac{\alpha}{s^{\alpha}}\right) \mathscr{L}_{x} \mathscr{L}_{t}\left\{\phi_{0 x x}\right\}\right]-c \mathscr{L}_{x}^{-1} \mathscr{L}_{t}^{-1}\left[\left(1-\alpha+\frac{\alpha}{s^{\alpha}}\right) \mathscr{L}_{x} \mathscr{L}_{t}\left\{\phi_{0}\right\}\right] \\
& -b \mathscr{L}_{x}^{-1} \mathscr{L}_{t}^{-1}\left[\left(1-\alpha+\frac{\alpha}{s^{\alpha}}\right) \mathscr{L}_{x} \mathscr{L}_{t}\left\{A_{0}\right\}\right]+\mathscr{L}_{x}^{-1} \mathscr{L}_{t}^{-1}\left[\left(1-\alpha+\frac{\alpha}{s^{\alpha}}\right) \mathscr{L}_{x} \mathscr{L}_{t}\{h(x, t)\}\right] \\
\phi_{2}= & \mathscr{L}_{x}^{-1} \mathscr{L}_{t}^{-1}\left[\left(1-\alpha+\frac{\alpha}{s^{\alpha}}\right) \mathscr{L}_{x} \mathscr{L}_{t}\left\{\phi_{1 x x}\right\}\right]-c \mathscr{L}_{x}^{-1} \mathscr{L}_{t}^{-1}\left[\left(1-\alpha+\frac{\alpha}{s^{\alpha}}\right) \mathscr{L}_{x} \mathscr{L}_{t}\left\{\phi_{1}\right\}\right] \\
& -b \mathscr{L}_{x}^{-1} \mathscr{L}_{t}^{-1}\left[\left(1-\alpha+\frac{\alpha}{s^{\alpha}}\right) \mathscr{L}_{x} \mathscr{L}_{t}\left\{A_{1}\right\}\right], \\
\phi_{3}= & \mathscr{L}_{x}^{-1} \mathscr{L}_{t}^{-1}\left[\left(1-\alpha+\frac{\alpha}{s^{\alpha}}\right) \mathscr{L}_{x} \mathscr{L}_{t}\left\{\phi_{2 x x}\right\}\right]-c \mathscr{L}_{x}^{-1} \mathscr{L}_{t}^{-1}\left[\left(1-\alpha+\frac{\alpha}{s^{\alpha}}\right) \mathscr{L}_{x} \mathscr{L}_{t}\left\{\phi_{2}\right\}\right] \\
& -b \mathscr{L}_{x}^{-1} \mathscr{L}_{t}^{-1}\left[\left(1-\alpha+\frac{\alpha}{s^{\alpha}}\right) \mathscr{L}_{x} \mathscr{L}_{t}\left\{A_{2}\right\}\right] .
\end{aligned}
$$

The final solution can be written as

$$
\phi(x, t)=\sum_{n=0}^{\infty} \phi_{n}(x, t) .
$$

Equations (22) and (26) are the general series solutions of equation (1) in both $\mathrm{CF}$ and $\mathrm{ABC}$ sense.

\section{Convergence of MDLDM for the Proposed Model}

Here, we discuss the convergence of the proposed method for the considered model equation (1). For this, we consider equation (1) in the operator form:

$$
\mathscr{T}(\phi)=\frac{\partial^{2} \phi}{\partial t^{2}}=\frac{\partial^{2} \phi}{\partial x^{2}}-c \phi-b g(\phi)+h(x, t) .
$$

Let $H \in L^{2}(\mathscr{T}) \forall \phi \in H$, where $H=\mathscr{L}_{\phi}^{2}[(m, n) \times[0, T]]$ [65], such that

$$
\phi:=[(m, n) \times[0, T]] \longrightarrow \mathbb{R}^{2},
$$

with $\quad m \ll 0$ and $B=[(m, n) \times[0, T]] \quad$ where $\|\phi\|_{H}^{2}=\int_{B} \phi^{2} \mathrm{~d} x \mathrm{~d} t$, then

$$
\mathscr{L}_{x}^{-1} \mathscr{L}_{t}^{-1}\left\{\mathscr{L}_{x} \mathscr{L}_{t}\{\phi(x, t)\}\right\}<\infty .
$$

For the operator $\mathscr{T}$ to be hemicontinuous [65], we consider the hypothesis as follows.

Hypothesis 1

$H_{1}:\langle\mathscr{T}(\phi)-\mathscr{T}(\psi), \phi-\psi\rangle \geq \kappa_{1}\|\phi-\psi\|^{2}, \phi, \psi \in H$ and $\kappa_{1}>0$.

$H_{2}$ : for $q>0$, there exists a constant $C(q)>0$, and $\forall \phi, \psi \in H$, with $\|\phi+\psi\|^{2} \leq q$, we obtain $\langle\mathscr{T}(\phi)-\mathscr{T}(\psi)\rangle \leq c(m)\|\phi-\psi\|, \forall \phi, \psi \in H$.

Theorem 1 (sufficient conditions of convergence). The proposed method is applied to equation (1) without initial and boundary conditions, converging to a particular solution.

Here, we use hypothesis 1 for operator $\mathscr{T}(\phi)$ in equation (1), such that

$$
\begin{aligned}
\mathscr{T}(\phi)-\mathscr{T}(\psi) & =\frac{\partial^{2} \phi}{\partial x^{2}}-\frac{\partial^{2} \psi}{\partial x^{2}}-c \phi+c \psi-b g(\phi)+-b g(\psi)+h-h, \\
& =\frac{\partial^{2}}{\partial x^{2}}(\phi-\psi)-c(\phi-\psi)-b g(\phi-\psi) .
\end{aligned}
$$

On taking the inner product, we obtain

$$
\langle\mathscr{T}(\phi)-\mathscr{T}(\psi), \phi-\psi\rangle=\left\langle\frac{\partial^{2}}{\partial x^{2}}(\phi-\psi), \phi-\psi\right\rangle-c\langle\phi-\psi, \phi-\psi\rangle-b g\langle\phi-\psi, \phi-\psi\rangle,
$$

when $g(\phi)=\phi^{2}$, then the above equation can be written as 


$$
\langle\mathscr{T}(\phi)-\mathscr{T}(\psi), \phi-\psi\rangle=\left\langle\frac{\partial^{2}}{\partial x^{2}}(\phi-\psi), \phi-\psi\right\rangle-c\langle\phi-\psi, \phi-\psi\rangle-b\left\langle(\phi-\psi)^{2},(\phi-\psi)^{2}\right\rangle,
$$

and we can put conditions on the operator $\partial^{2} / \partial x^{2}$ in $H$, such that for $\eta>0$, we can define

$$
\begin{aligned}
& \frac{\partial^{2}}{\partial^{2} x}(\phi-\psi) \leq \eta\|\phi-\psi\|^{2}, \\
& \langle\mathscr{T}(\phi)-\mathscr{T}(\psi), \phi-\psi\rangle \\
& \leq \eta\|\phi-\psi\|^{2}-c\|\phi-\psi\|^{2}-b\left\|(\phi-\psi)^{2}\right\|^{2} \\
& =\eta\|\phi-\psi\|^{2}-c\|\phi-\psi\|^{2}-b\|\phi+\psi\|^{2}\|\phi-\psi\|^{2} \\
& \langle\mathscr{T}(\phi)-\mathscr{T}(\psi), \phi-\psi\rangle \leq(\eta-c-b \sigma)\|\phi-\psi\|^{2} .
\end{aligned}
$$

Taking $\kappa_{1}=(\eta-c-b \sigma)>0$, we can write

$$
\langle\mathscr{T}(\phi)-\mathscr{T}(\psi), \phi-\psi\rangle \leq \kappa_{1}\|u-w\|^{2} .
$$

Hence, hypothesis 1 is satisfied.

Next, we verify hypothesis 1 for operator $\mathscr{T}(\phi)$.

For every $q>0$, there exists a constant $C(q)>0$ such that for $\phi, \psi \in \mathrm{H}$ with $\|\phi+\psi\|^{2} \leq q$, we have

$$
\langle\mathscr{T}(\phi)-\mathscr{T}(\psi)\rangle \leq C(q)\|\phi-\psi\|, \quad \forall \phi, \psi \in H .
$$

Now, for the proof, considering $\varpi_{1} \in H$, we have

$$
\left\langle\mathscr{T}(\phi)-\mathscr{T}(\psi), \varpi_{1}\right\rangle=\left\langle\frac{\partial^{2}}{\partial x^{2}}(\phi-\psi), \varpi_{1}\right\rangle-c\left\langle\phi-\psi, \varpi_{1}\right\rangle-b\left\langle(\phi-\psi)^{2}, \varpi_{1}^{2}\right\rangle
$$

By applying Cauchy-Schwartz inequality and since $\phi$ and $\psi$ are bounded, we have

$$
\begin{aligned}
& {\left[\frac{\partial^{2}}{\partial^{2} x}(\phi-\psi), \Phi_{1}\right] \leq \eta_{1}\|\phi-\psi\| \leq \eta_{1}\|\phi-\psi\|\left\|\omega_{1}\right\|,} \\
& c\left[-\left((\phi-\psi), ळ_{1}\right)\right] \leq c\|\phi-\psi\| \leq c\|\phi-\psi\|\left\|\varpi_{1}\right\|, \\
& b\left[-\left((\phi-\psi)^{2}, \varpi_{1}^{2}\right)\right] \leq b\|\phi+\psi\|\|\phi-\psi\| \leq b q\|\phi-\psi\|\left\|\varpi_{1}\right\| .
\end{aligned}
$$

$$
\left\langle\mathscr{T}(\phi)-\mathscr{T}(\psi), \varpi_{1}\right\rangle \leq C(q)\|\phi-\psi\|\left\|\varpi_{1}\right\|, \quad \text { where } C(q)=\left(\eta_{1}+c+b q\right)>0 \text {. }
$$

$$
\begin{aligned}
\phi(x, 0) & =B \tan (\Omega x), \\
\phi_{t}(x, 0) & =B \varepsilon \Omega \sec ^{2}(\Omega x), \quad 0 \leq x \leq 2,
\end{aligned}
$$

where

$$
\begin{aligned}
& B=\sqrt{\frac{\zeta}{\gamma},} \\
& \Omega=\sqrt{\frac{-\zeta}{2\left(\sigma+\epsilon^{2}\right)}} .
\end{aligned}
$$

Therefore, we can write linear $\mathrm{KG}$ equation

$$
\begin{aligned}
D_{t}^{\alpha} \phi-c \frac{\partial^{2} \phi}{\partial x^{2}}+b \phi^{3} & =0, \quad 1<\alpha \leq 2, \\
g(\phi) & =\phi^{3}, \\
h(x, t) & =0,
\end{aligned}
$$

with $b=c=1$. The subsidiary conditions are given as

Here, we consider numerical examples of the nonlinear TFKG equation and discuss two cases.

$$
\text { as }
$$


Case I: consider the TFKG equation (39) in Caputo-Fabrizio's sense as

$$
{ }^{\mathrm{CF}} D_{t}^{\alpha+m} \phi(x, t)-\frac{\partial^{2} \phi}{\partial x^{2}}+\phi^{3}(x, t)=0, \quad 0<\alpha \leq 1, m=1 .
$$

The approximate solution of equation (43) by the techniques discussed in Section 3 is obtained as

$$
\begin{aligned}
\phi_{0}= & B \tan (\Omega x)+t B \varepsilon \Omega \sec ^{2}(\Omega x) \\
\phi_{1}= & \left((1-\alpha) t+\frac{\alpha t^{2}}{2 !}\right)\left[2 B \Omega^{2} \sec ^{2}(\Omega x) \tan (\Omega x)-B^{2} \tan ^{3}(\Omega x)\right] \\
& +B \epsilon \Omega\left(\frac{(1-\alpha) t^{2}}{2 !}+\frac{\alpha t^{3}}{3 !}\right)\left[4 \Omega^{2} \sec ^{2}(\Omega x) \tan ^{2}(\Omega x)+2 \Omega^{2} \sec ^{4}(\Omega x)-3 B^{2} \tan ^{2}(\Omega x) \sec ^{2}(\Omega x)\right] \\
& -6 B^{3} \Omega^{2} \epsilon^{2}\left(\frac{(1-\alpha) t^{3}}{3 !}+\frac{\alpha t^{4}}{4 !}\right)\left[\sec ^{4}(\Omega x) \tan (\Omega x)\right] \\
& -6 B^{3} \Omega^{3} \epsilon^{3}\left(\frac{(1-\alpha) t^{4}}{4 !}+\frac{\alpha t^{5}}{5 !}\right)\left[\sec h^{6}(\Omega x)\right] .
\end{aligned}
$$

The final solution in the series form up to $\mathcal{O}(2)$ is given by

$$
\phi(x, t)=\phi_{0}+\phi_{1}
$$

Case II: similar to the previous section, the TFKG equation (39) in Atangana-Baleanu-Caputo's sense,

$$
{ }^{\mathrm{ABC}} D_{t}^{\alpha} \phi(x, t)-\frac{\partial^{2} \phi}{\partial x^{2}}+\phi^{3}(x, t)=0, \quad 1<\alpha \leq 2 .
$$

The solution of equation (46) is obtained as

$$
\begin{aligned}
\phi_{0}= & B \tan (\Omega x)+t B \varepsilon \Omega \sec ^{2}(\Omega x) \\
\phi_{1}= & \left((1-\alpha)+\frac{\alpha t^{\alpha}}{\Gamma(\alpha+1)}\right)\left[2 B \Omega^{2} \sec ^{2}(\Omega x) \tan (\Omega x)-B^{2} \tan ^{3}(\Omega x)\right] \\
& +B \epsilon \Omega\left((1-\alpha) t+\frac{\alpha t^{\alpha+1}}{\Gamma(\alpha+2)}\right)\left[4 \Omega^{2} \sec ^{2}(\Omega x) \tan ^{2}(\Omega x)+2 \Omega^{2} \sec ^{4}(\Omega x)-3 B^{2} \tan ^{2}(\Omega x) \sec ^{2}(\Omega x)\right] \\
& -6 B^{3} \Omega^{2} \epsilon^{2}\left(\frac{(1-\alpha) t^{2}}{2 !}+\frac{\alpha t^{\alpha+2}}{\Gamma(\alpha+3)}\right)\left[\sec ^{4}(\Omega x) \tan (\Omega x)\right] \\
& -6 B^{3} \Omega^{3} c^{3}\left(\frac{(1-\alpha) t^{3}}{3 !}+\frac{\alpha t^{\alpha+3}}{\Gamma(\alpha+4)}\right)\left[\sec ^{6}(\Omega x)\right] .
\end{aligned}
$$

The final solution in series form up to $\mathcal{O}(2)$ is

$$
\phi(x, t)=\phi_{0}+\phi_{1}
$$

5.2. Discussion. For numerical illustrations, we have considered the parameters as $\zeta=-1, \gamma=1, \sigma=-8.5$, and $\epsilon$ $=0.05$. The numerical solutions, equations (45) and (48), and exact solution equation (42) associated with the Caputo-Fabrizio's (CF) sense and Atangana-Baleanu-Caputo's (ABC) sense are depicted in Figure 1(a), with variation in the time-fractional coefficient $(\alpha)$. One can see that TFKG equation (39) may admit the excitation of monotonic shocks in an inviscid dynamical system. This degree enhancement in $\alpha$ suppresses the wave amplitude as it affects the nonlinearity/dispersion effects. To 

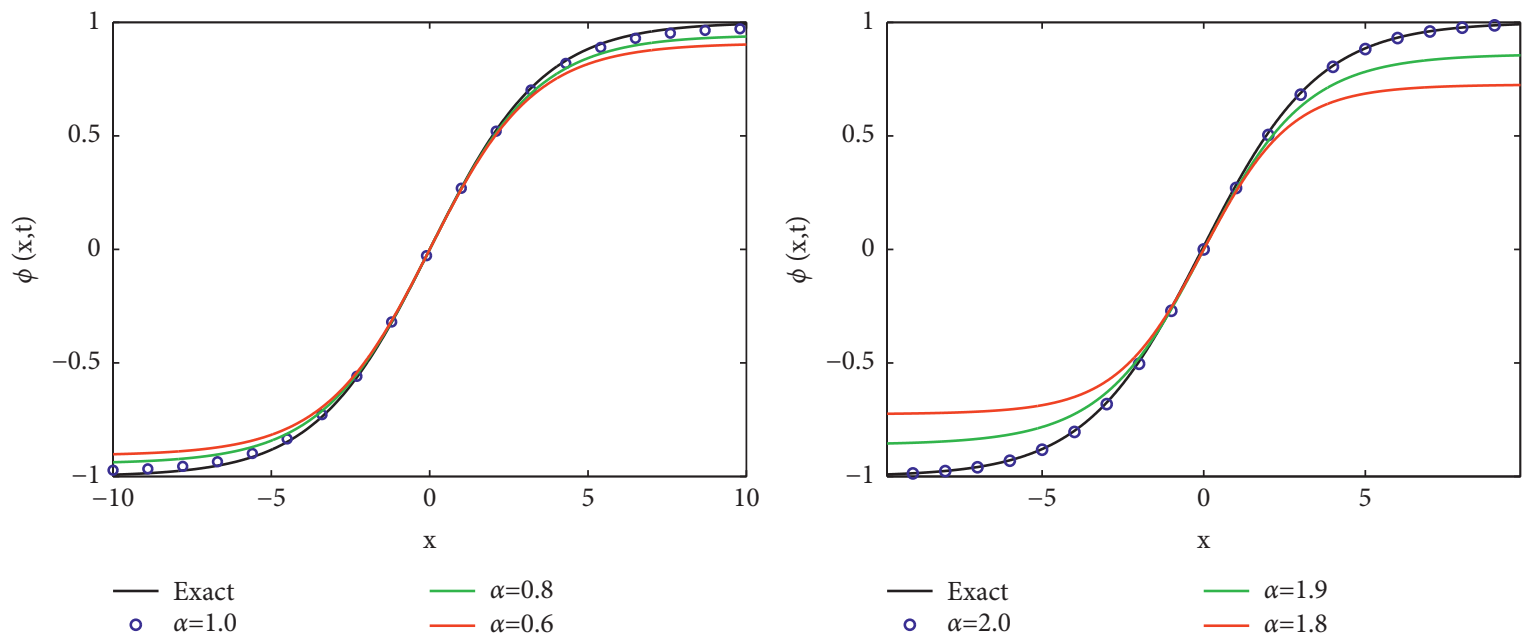

(a)
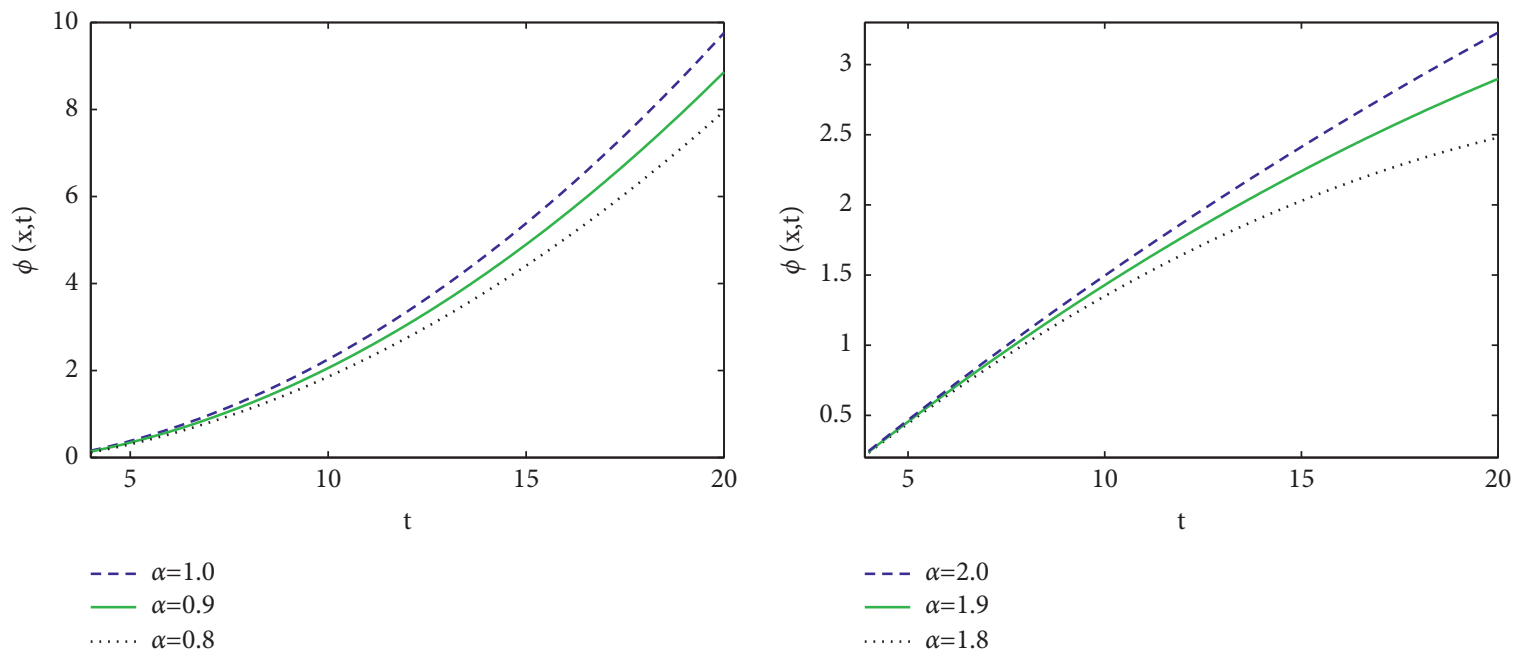

(b)

Figure 1: (a) The comparison between equations (42), (45), and (48) for different values of $\alpha$; (b) solution profiles of $\phi(x, t)$ against $t$ (time) for a variety of $\alpha$, as mentioned in the plots.

see the effect of a temporal variable $(t)$ on the wave solutions, equations (45) and (48) are displayed in Figure 1(b); it reveals that $\phi(x, t)$ rises with time. The three-dimensional profiles for equations (45) and (48) are shown versus $x$ and $t$ in Figure 2. Figure 2(a) represents the physical behavior of equation (45) for $\alpha=1$, while Figure 2(b) represents equation (48) for $\alpha=2$. It reveals the evolution of localized shock excitations. We have depicted the solution equation (45) versus $x$ when $t=0$ (solid black curve), 0.4 (circles), 0.6 (solid green curve), and 0.8 (dotted curve) in Figure 3(a), with $\alpha=1$ and 0.8 , respectively. Obviously, the wave amplitude enhances with variations in $t$. By choosing $\alpha=2$ and 1.9, we have illustrated equation (48) in Figure 3(b). It infers that coefficient $(\alpha)$ significantly increases the wave amplitudes.
5.3. Example. Consider a nonlinear TFKG equation

$$
D_{t}^{\alpha} \phi-\frac{\partial^{2} \phi}{\partial x^{2}}+\frac{3}{4} \phi-\frac{3}{2} \phi^{3}=0, \quad 1<\alpha \leq 2,
$$

with $c=3 / 4, b=3 / 2, g(\phi)=\phi^{3}, h(x, t)=0$, and

$$
\begin{aligned}
\phi(x, 0) & =-\sec h(x), \\
\phi_{t}(x, 0) & =\frac{1}{2} \sec h(x) \tanh (x) .
\end{aligned}
$$

The exact solution for $\alpha=2$ of the above equation is [30]

$$
\phi(x, t)=-\sec h\left(x+\frac{t}{2}\right) .
$$




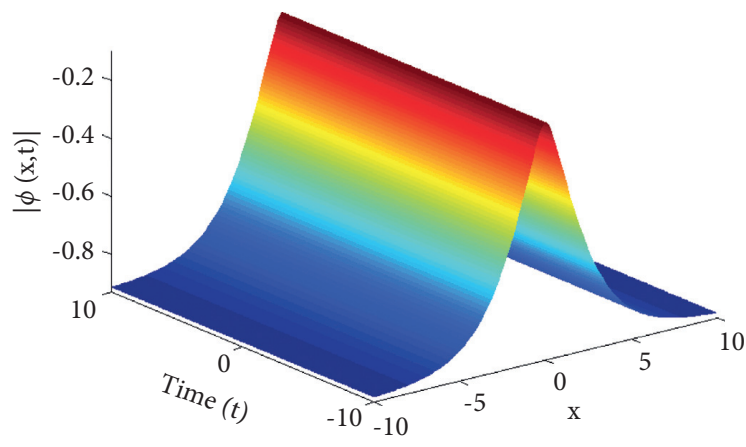

(a)

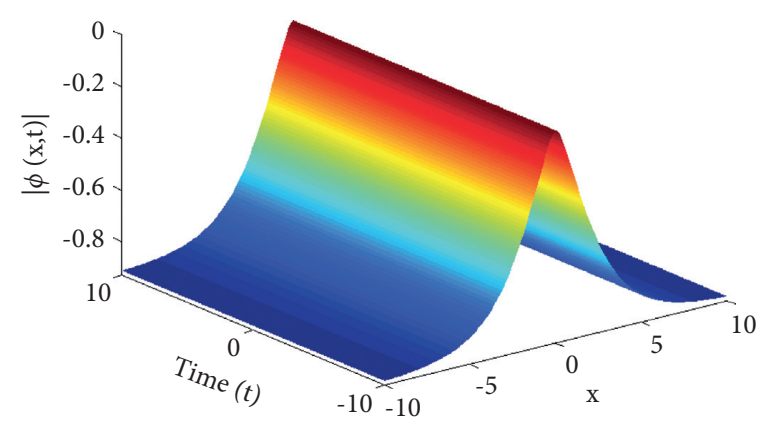

(b)

Figure 2: The surface plots for CF equation (45) solution and $\mathrm{ABC}$ solution equation (48) for the parameters used in Figure 1(a).
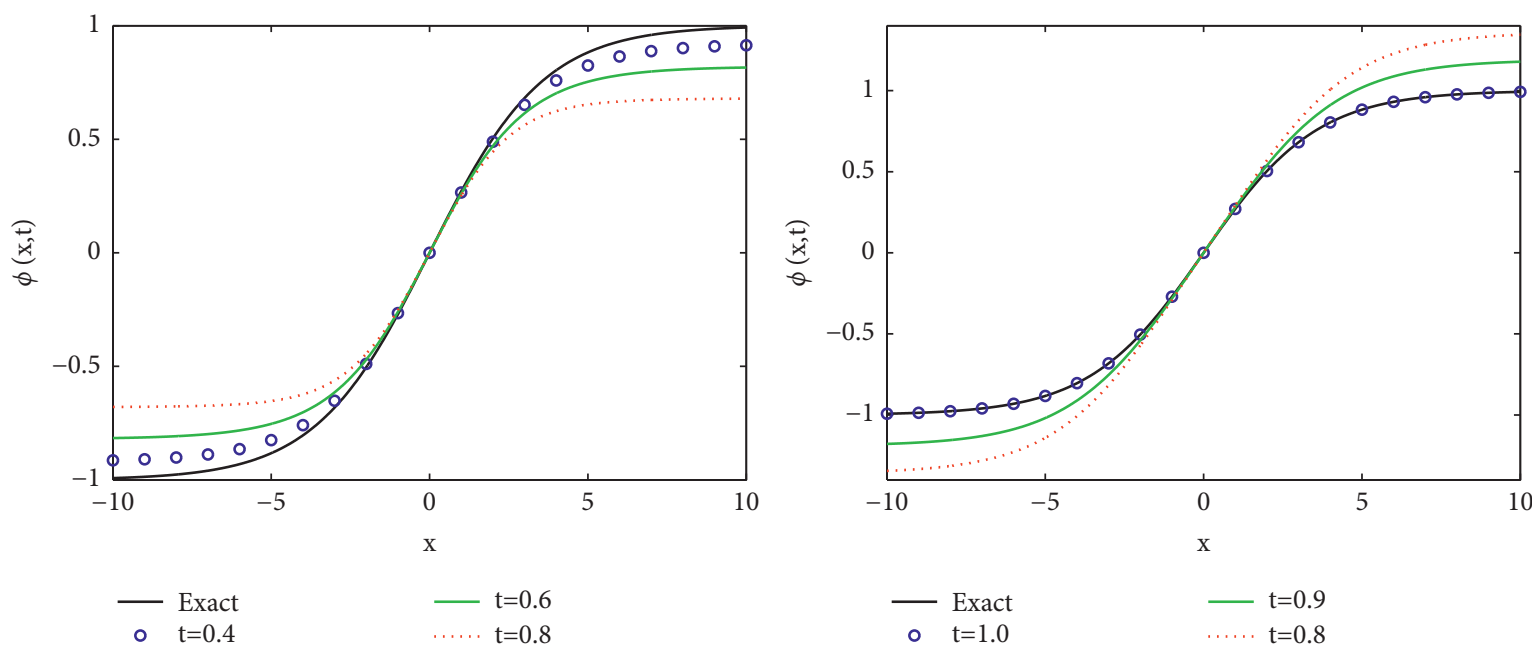

(a)
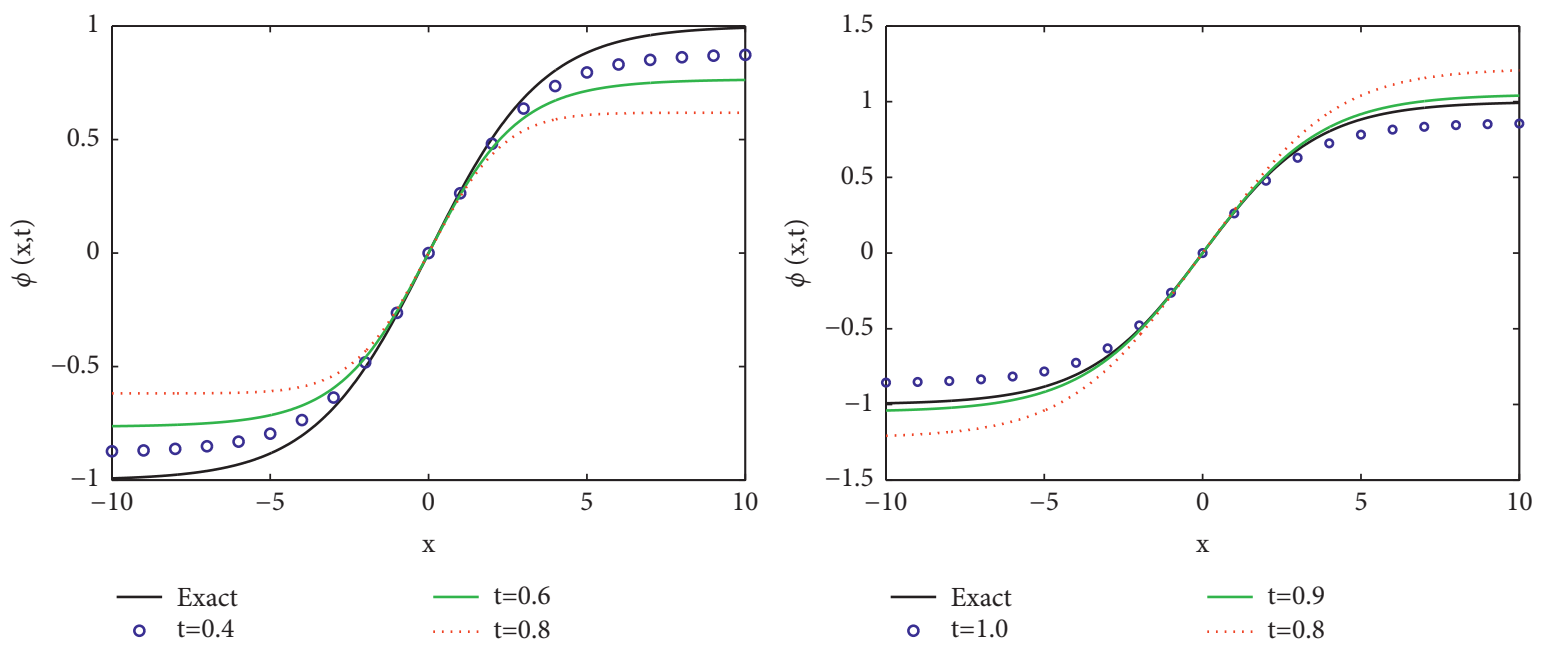

(b)

FIgURE 3: The solution profiles of $|\phi(x, t)|$ versus different values of $t$. 
Case I: consider the TFKG equation (49) in Caputo-Fabrizio's sense as

$$
{ }^{C F} D_{t}^{\alpha+m} \phi-\frac{\partial^{2}}{\partial x^{2}} \phi+\frac{3}{4} \phi-\frac{3}{2} \phi^{3}=0, \quad 0<\alpha \leq 1, m=1 .
$$

The approximate solution of equation (51) together with initial conditions (50) by the techniques discussed in Section 3 is obtained as

$$
\begin{aligned}
& \begin{aligned}
\phi_{0}= & \frac{t}{2} \sec h(x) \tanh (x)-\sec h(x), \\
\phi_{1}= & (1-\alpha+\alpha t)\left[\sec h^{3}(x)-\tanh ^{2}(x) \sec h(x)+\frac{3}{4} \sec h(x)-\frac{3}{2} \sec h^{3}(x)\right] \\
& +\left((1-\alpha) t+\frac{\alpha t^{2}}{2 !}\right)\left[\tanh ^{3}(x) \sec h(x)-5 \sec h^{3}(x) \tanh (x)+\frac{3}{8} \sec h(x) \tanh (x)+\frac{9}{4} \sec h^{3}(x) \tanh (x)\right] \\
& -\left(\frac{(1-\alpha) t^{2}}{2 !}+\frac{\alpha t^{3}}{3 !}\right)\left[\frac{9}{8} \sec h^{3}(x) \tanh (x)\right]+\left(\frac{(1-\alpha) t^{3}}{3 !}+\frac{\alpha t^{4}}{4 !}\right)\left[\frac{3}{16} \sec ^{3}(x) \tanh ^{3}(x)\right] .
\end{aligned} \\
& \text { The final solution in series form up to } \mathcal{O}(2) \text { is given by } \quad{ }^{A B C} D_{t}^{\alpha} \phi-\frac{\partial^{2}}{\partial x^{2}} \phi+\frac{3}{4} \phi-\frac{3}{2} \phi^{3}=0, \quad 1<\alpha \leq 2 .
\end{aligned}
$$

$$
\phi(x, t)=\phi_{0}+\phi_{1} .
$$

Case II: consider the TFKG equation (49) in Atangana-Baleanu-Caputo's sense

We obtain the approximate solution in equation (52) together with initial condition (50) and using the techniques discussed in Section 3:

$$
\begin{aligned}
\phi_{0}= & \frac{t}{2} \sec h(x) \tanh (x)-\sec h(x) \\
\phi_{1}= & (1-\alpha+\alpha t)\left[\sec h^{3}(x)-\tanh ^{2}(x) \sec h(x)+\frac{3}{4} \sec h(x)-\frac{3}{2} \sec h^{3}(x)\right] \\
& +\left((1-\alpha) t+\frac{\alpha t^{\alpha+1}}{\Gamma(\alpha+2)}\right)\left[\tanh ^{3}(x) \sec h(x)-5 \sec h^{3}(x) \tanh (x)+\frac{3}{8} \sec h(x) \tanh (x)+\frac{9}{4} \sec h^{3}(x) \tanh (x)\right] \\
& -\left(\frac{(1-\alpha) t^{2}}{2 !}+\frac{\alpha t^{\alpha+2}}{\Gamma(\alpha+3)}\right)\left[\frac{9}{8} \sec ^{3}(x) \tanh ^{2}(x)\right]+\left(\frac{(1-\alpha) t^{3}}{3 !}+\frac{\alpha t^{\alpha+3}}{\Gamma(\alpha+4)}\right)\left[\frac{3}{16} \sec h^{3}(x) \tanh ^{3}(x)\right],
\end{aligned}
$$

The final solution in series form up to $\mathcal{O}(2)$ is given by

$$
\phi(x, t)=\phi_{0}+\phi_{1}
$$

5.4. Discussion. Figure 4(a) displays the absolute of wave solution equations (54) and (57), having variations in $(\alpha)$ with $t=0.3$ and $t=1$, respectively, with exact solution given in equation (51). Notice that the numerical solutions, CF equation (54), and $\mathrm{ABC}$ equation (57) exactly match to the exact solution equation (51). We observe that TFKG admits pulse-shaped solitons. We also know that the solution equations (54) and (57) in Figure 4(b) reveal that the amplitude of the solitary potentials goes up as $t$ rises. The three-dimensional profiles for equations (54) and (57) are shown versus $x$ and $t$ in Figure 5. Figure 5(a) represents the physical behavior of equation (54) for $\alpha=1$, while Figure 5(b) 1 represents equation (57) for $\alpha=2$. It reveals the evolution of localized shock excitations. We have depicted the solution equation (54) versus $x$ with $t=0.6$ (dashed line), 0.4 (solid curve), and 0.2 (dotted curve) in Figure 6(a), when $\alpha=1$ and 0.7 , respectively. Obviously, the wave amplitude enhances with variations in $t$. By choosing $\alpha=2$ and 1.7, we have illustrated equation (57) in Figure 6(b). It infers that coefficient $(\alpha)$ significantly increases the wave amplitudes. 

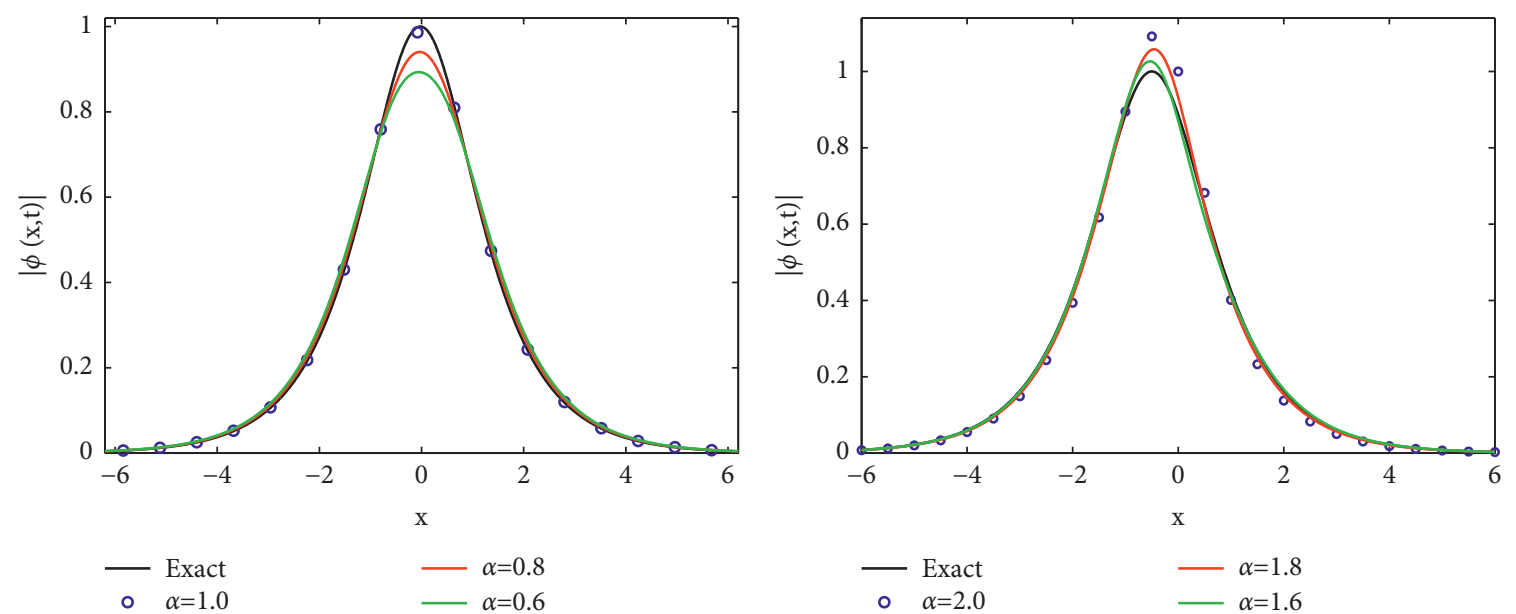

(a)
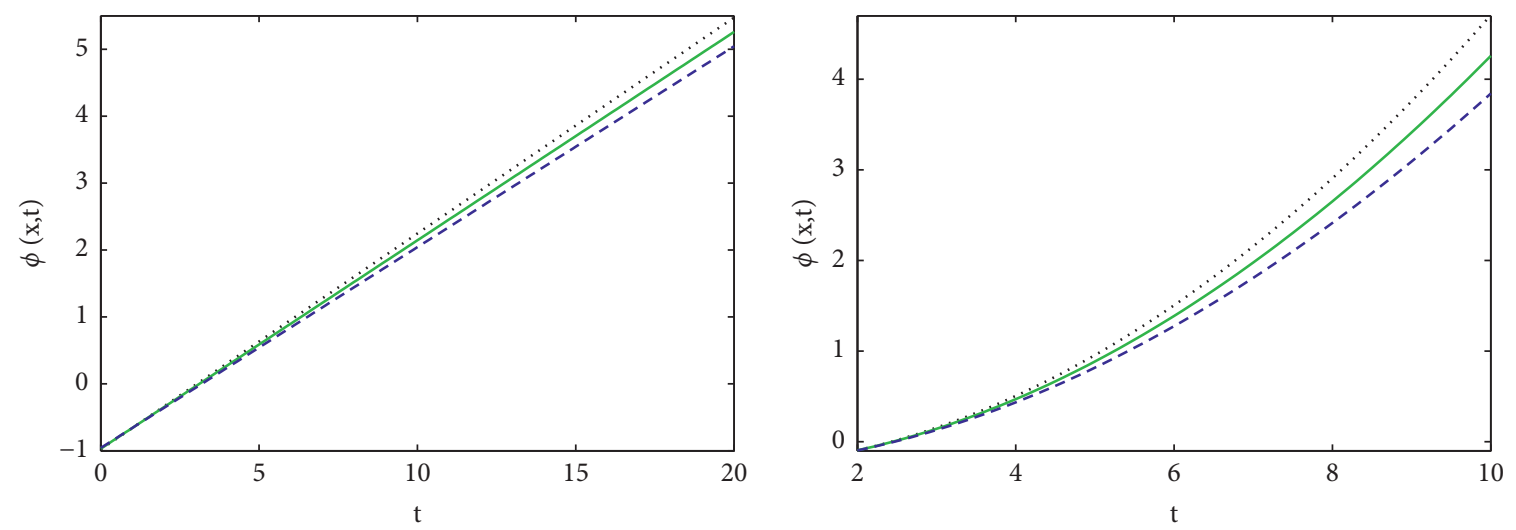

$$
\begin{aligned}
\alpha=1.00 \\
-\alpha=0.95
\end{aligned}
$$$$
\text { … } \alpha=2.00
$$$$
-\alpha=1.95
$$$$
--\alpha=1.90
$$

(b)

Figure 4: (a) The comparison between equations (50), (52), and (54) for different values of alpha; (b) solution profiles of $\phi(x, t)$ vs time $t$ for different values of $\alpha$.

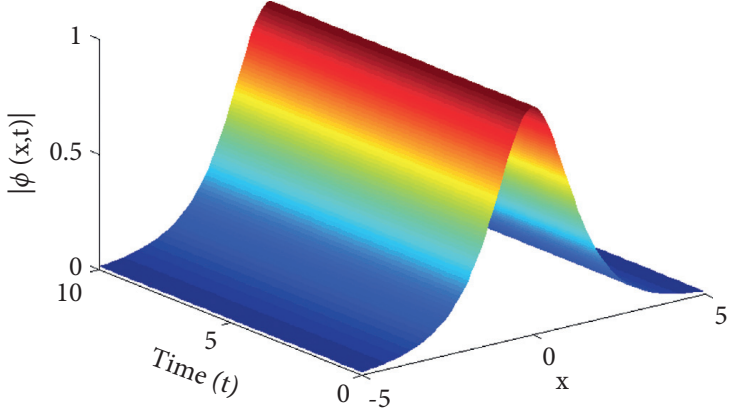

(a)

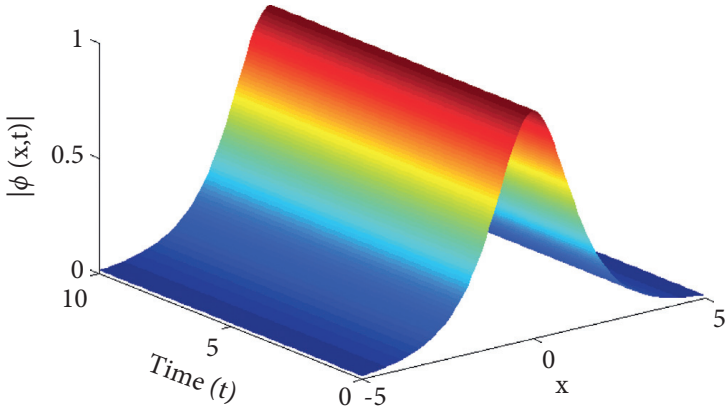

(b)

FIgURE 5: The surface plots of solution of TFKG with CF and ABC operators for the parameters used in Figure 4(a). 

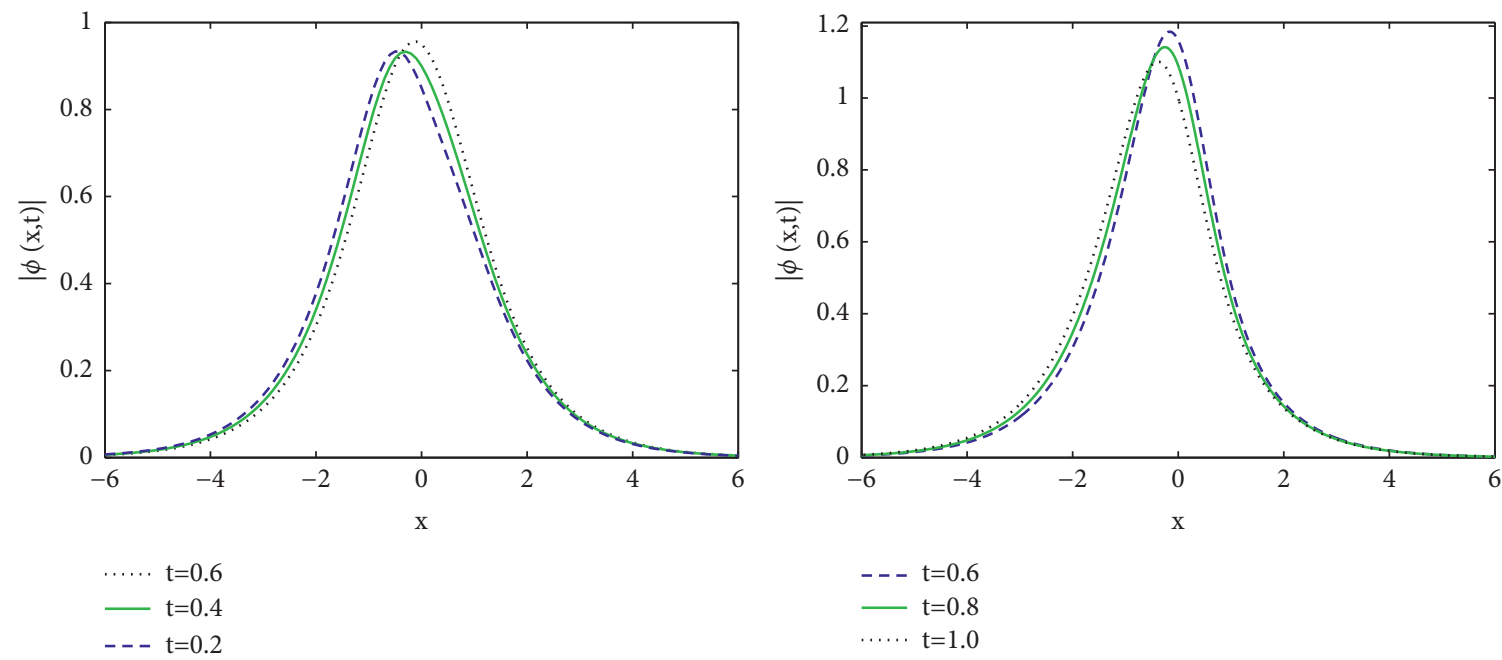

(a)
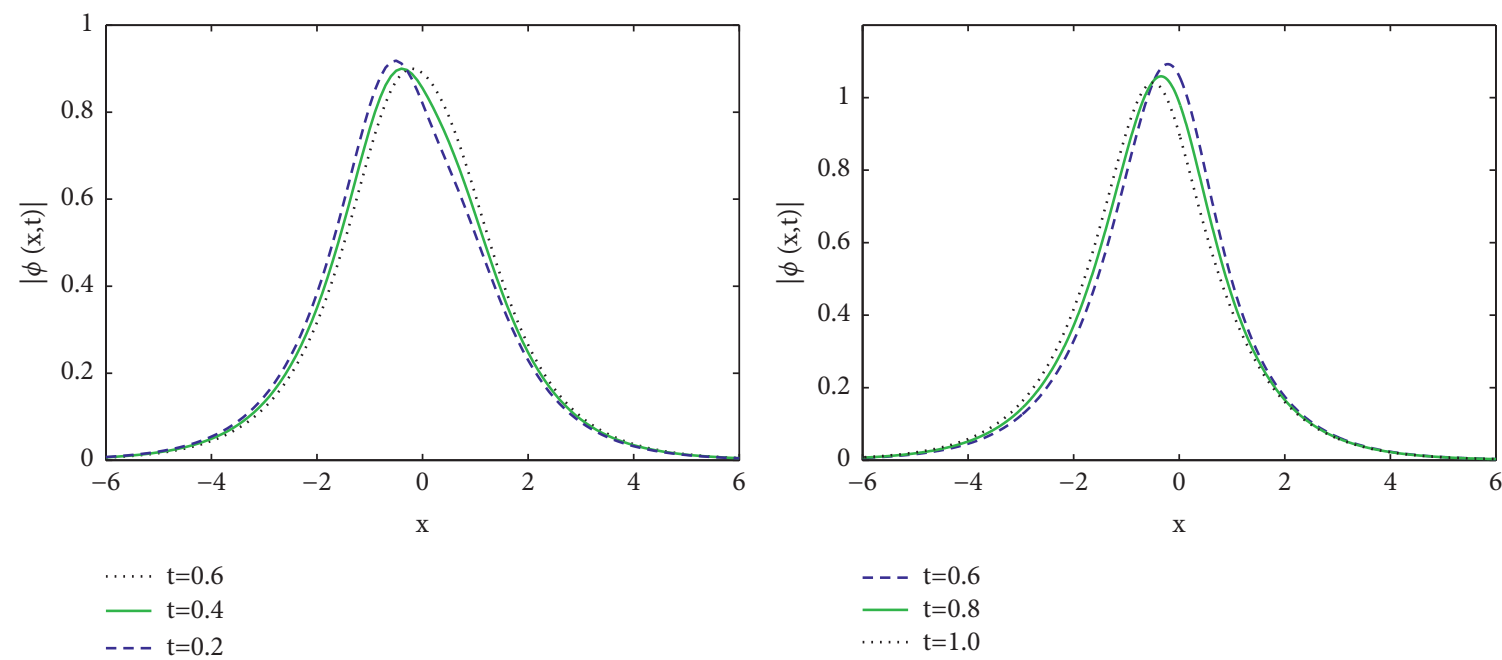

(b)

Figure 6: Solution profiles of $|\phi(x, t)|$ for different values of $\alpha$ with different values of time $t$.

\section{Conclusion}

We have studied the time-fractional Klein-Gordon equation using the MDLD method. The approximate solutions of nonlinear Klein-Gordon equation are obtained in the form of the series. It is very important to notice that even after some iterations, more accurate results are obtained. It is perceived that our proposed method provides accurate numerical results without perturbation and discretization for nonlinear differential equations with fractional operators. The numerical results obtained for particular examples are compared with the exact solutions at the classical order. The effect of the altered fractional orders of the considered numerical illustrations is shown explicitly, where good agreements are obtained. The Klein-Gordon equation is evidently a nonlinear PDE and thus a perfect model for understanding the nonlinearity/dispersion effects and the evolution of localized shock excitations. It is inferred in this manuscript that fractional order significantly increases the wave amplitudes. Similar to the Klein-Gordon equation, the Sine-Gordon equation bears kink-anti-kink phenomena. The Sine-Gordon potential has unbounded minimum points; however, two of them are assumed in the kink solutions of the system. By considering $u \simeq \sin u$, the considered system becomes the Sine-Gordon equation. In future work, it will be interesting to investigate the Sine-Gordon model with nonlinear AC/DC drives 
with different fractional operators to study the solitonic behavior, localized modes in single and in stacked long Josephson junctions with a variety of potentials, parity time symmetry, the nonlinearity/dispersion effects, and evolution of the localized monotonic shocks [67-73].

\section{Data Availability}

The data that support the findings of this study are available on request to the corresponding author.

\section{Conflicts of Interest}

All the authors have no conflicts of interest regarding this article.

\section{Authors' Contributions}

All the authors have equal contributions in the manuscript. Furthermore, the authors have checked and approved the final version of the manuscript.

\section{References}

[1] K. B. Oldham and J. Spanier, The Fractional Calculus, Academic, New York, NY, USA, 1974.

[2] I. Podlubny, Fractional Differential Equations, Academic, New York, NY, USA, 1999.

[3] R. Hilfer, Application of Fractional Calculus in Physics, World Scientific, Singapore, 2000.

[4] R. L. Magin and M. Ovadia, "Modeling the cardiac tissue electrode interface using fractional calculus," Journal of $\mathrm{Vi}$ bration and Control, vol. 14, no. 9-10, pp. 1431-1442, 2008.

[5] L. Sommacal, P. Melchior, A. Oustaloup, J.-M. Cabelguen, and A. J. Ijspeert, "Fractional multi-models of the frog gastrocnemius muscle," Journal of Vibration and Control, vol. 14, no. 9-10, pp. 1415-1430, 2008.

[6] L. Zhang, M. ur Rahman, M. Arfan, and A. Ali, "Investigation of mathematical model of transmission co-infection TB in HIV community with a non-singular kernel," Results in Physics, vol. 28, Article ID 104559, 2021.

[7] B. T. Krishna and K. V. V. S. Reddy, "Active and passive realization of fractance device of order 1/2," Active and Passive Electronic Components, vol. 2008, Article ID 369421, 5 pages, 2008.

[8] Y. Pu, X. Yuan, K. Liao et al., "A recursive two-circuits series analog fractance circuit for any order fractional calculus," Optical Information Processing of Proceedings of SPIE, vol. 6027, pp. 509-519, 2006.

[9] N. Heymans, "Dynamic measurements in long-memory materials: fractional calculus evaluation of approach to steady state," Journal of Vibration and Control, vol. 14, no. 9-10, pp. 1587-1596, 2008.

[10] M. F. M. Lima, J. A. T. Machado, J. A. T. Machado, and M. Crisóstomo, "Experimental signal analysis of robot impacts in a fractional calculus perspective," Journal of Advanced Computational Intelligence and Intelligent Informatics, vol. 11, no. 9, pp. 1079-1085, 2007.

[11] R. Panda and M. Dash, "Fractional generalized splines and signal processing," Signal Processing, vol. 86, no. 9, pp. 2340-2350, 2006.
[12] G. W. Bohannan, "Analog fractional order controller in temperature and motor control applications," Journal of Vibration and Control, vol. 14, no. 9-10, pp. 1487-1498, 2008.

[13] S. S. Ray, A. Atangana, S. C. O. Noutchie, M. Kurulay, N. Bildik, and A. Kilicman, "Fractional calculus and its applications in applied mathematics and other sciences," Mathematical Problems in Engineering, vol. 2014, Article ID 849395, 2 pages, 2014.

[14] M. Al-Smadi, A. Freihat, H. Khalil, S. Momani, and R. A. Khan, "Numerical multistep approach for solving fractional partial differential equations," International Journal of Computational Methods, vol. 14, no. 3, 2017.

[15] G. Walter. Der, "Comptoneffekt nach der schrödingerschen theorie," Zeitschrift für Physik, vol. 40, pp. 1-2, 1926.

[16] J. A. T. Machado, "A probabilistic interpretation of the fractional-order differentiation," Fractional Calculus and Applied Analysis, vol. 6, pp. 73-80, 2003.

[17] K. Diethelm, "An algorithm for the numerical solution of differential equations of fractional order," Electronic Transactions on Numerical Analysis, vol. 5, p. 1, 1997.

[18] K. Diethelm and N. J. Ford, "Analysis of fractional differential equations," Journal of Mathematical Analysis and Applications, vol. 265, no. 2, pp. 229-248, 2002.

[19] M. Al-Smadi and O. A. Arqub, "Computational algorithm for solving fredholm time-fractional partial integro differential equations of dirichlet functions type with error estimates," Applied Mathematics and Computation, vol. 342, pp. 280-294, 2019.

[20] X. J. Yang, D. Baleanu, and H. M. Srivastava, Local Fractional Integral Transforms and Their Applications, Academic Press, New York, NY, USA, 2015.

[21] D. Baleanu, K. Diethelm, E. Scalas, and J. J. Trujillo, Fractional Calculus; Models and Numerical Methods, World Scientific, New York, NY, USA, 2012.

[22] O. Klein, "Quantentheorie und fünfdimensionale relativitätstheorie,” Zeitschrift für Physik, vol. 37, 1926.

[23] E. Yusufoglu, "The variational iteration method for studying the Klein-Gordon equation," Applied Mathematics Letters, vol. 21, pp. 669-674, 2008.

[24] N. H. Sweilam, M. M. Khader, and A. M. S. Mahdy, "On the numerical solution for the linear fractional Klein-Gordon equation using Legendre pseudo-spectral method," International Journal of Mathematics and Computer Applications Research, vol. 2, pp. 1-10, 2012.

[25] E. Abuteen, A. Freihat, M. Al-Smadi, H. Khalil, and R. A. Khan, "Approximate series solution of nonlinear, fractional Klein-Gordon equations using fractional reduced differential transform method," Journal of Mathematics and Statistics, vol. 12, no. 1, pp. 23-33, 2016.

[26] W. Greiner, Relativistic Quantum Mechanics. Wave Equations, Springer-Verlag, Springer, Germany, 3rd edition, 2000.

[27] J. J. Sakurai, Advanced Quantum Mechanics, Addison-Wesley, Boston, MA, USA, 1967.

[28] S. Weinberg, The Quantum Theory of Fields, Cambridge University Press, Cambridge, UK, 2002.

[29] M. Kurulay, "Solving the fractional nonlinear Klein-Gordon equation by means of the homotopy analysis method," Advances in Difference Equations, vol. 187, pp. 1-8, 2012.

[30] B. Bülbül and M. Sezer, "A new approach to numerical solution of nonlinear Klein-Gordon equation," Mathematical Problems in Engineering, vol. 2013, 2013. 
[31] M. Caputo and M. Fabrizio, "A new definition of fractional derivative without singular kernel," Progress in Fractional Differentiation and Applications, vol. 1, pp. 73-85, 2015.

[32] A. Atangana and D. Baleanu, "New fractional derivatives with nonlocal and non-singular kernel; Theory and application to heat transfer model," Thermal Science, vol. 20, 2016.

[33] E. Hesameddini and F. Fotros, "Solution for time-fractional coupled Klein-Gordon Schrodinger equation using decomposition method," International Mathematical Forum, vol. 7, pp. 1047-1056, 2012.

[34] K. Moaddy, S. Momani, and I. Hashim, "The non-standard finite difference scheme for linear fractional PDEs in fluid mechanics," Computers \& Mathematics with Applications, vol. 61, no. 4, pp. 1209-1216, 2011.

[35] O. Abdulaziz, I. Hashim, and A. Saif, "Series solutions of timefractional PDEs by homotopy analysis method," Differential Equations and Nonlinear Mechanics, vol. 2008, Article ID 686512, 16 pages, 2008.

[36] K. D. Kucche and S. T. Sutar, "Analysis of nonlinear fractional differential equations involving Atangana-Baleanu-Caputo derivative," Chaos, Solitons \& Fractals, vol. 143, Article ID 110556, 2021.

[37] A. Ali, Z. Gul, W. A. Khan, S. Ahmad, and S. Zeb, "Investigation of fractional order sine-gordon equation using Laplace adomian decomposition method," Fractals, vol. 29, no. 5, 2021.

[38] D. J. Evans and H. Bulut, "A new approach to the gas dynamics equation: an application of the decomposition method," International Journal of Computer Mathematics, vol. 79, pp. 752-761, 2002.

[39] X. J. Yang, D. Baleanu, and W. P. Zhong, "Approximate solutions for diffusion equations on Cantor space-time," Proceedings of the Romanian Academy-Series A, vol. 14, pp. 127-133, 2013.

[40] K. Moaddy, A. Freihat, M. Al-Smadi, E. Abuteen, and I. Hashim, "Numerical investigation for handling fractionalorder Rabinovich-Fabrikant model using the multistep approach," Soft Computing, vol. 22, no. 3, pp. 773-782, 2018.

[41] L. Debnath, "The double Laplace transforms and their properties with applications to functional, integral and partial differential equations," International Journal of Algorithms, Computing and Mathematics, vol. 2, no. 2, pp. 223-241, 2016.

[42] K. Khan, Z. Khan, A. Ali, and M. Irfan, "Investigation of hirota equation: modified double Laplace decomposition method," Physica Scripta, vol. 96, 2021.

[43] D. G. Duffy, Transform Methods for Solving Partial Differential Equations, CRC Press, Florida, USA, 2004.

[44] M. Al-Smadi, "Simplified iterative reproducing kernel method for handling time-fractional BVPs with error estimation," Ain Shams Engineering Journal, vol. 9, no. 4, pp. 2517-2525, 2018.

[45] A. Atangana, "Extension of the modified homotopy perturbation method for attractor one-dimensional Keller-Segel equations," Applied Mathematical Modelling, vol. 39, pp. 2815-3182, 2015.

[46] X. J. Yang, H. M. Srivastava, and C. Cattani, "Local fractional homotopy perturbation method for solving fractal partial differential equations arising in mathematical physics," Romanian Reports in Physics, vol. 67, pp. 752-761, 2015.

[47] S. T. Demiray, H. Bulut, and F. B. M. Belgacem, "Sumudu transform method for analytical solutions of fractional type ordinary differential equations," Mathematical Problems in Engineering, vol. 2015, Article ID 131690, 6 pages, 2015.

[48] D. Baleanu, H. M. Srivastava, and X. J. Yang, "Local fractional variational iteration algorithms for the parabolic Fokker-
Planck equation defined on cantor sets," Progress in Fractional Differentiation and Applications, vol. 1, pp. 1-11, 2015.

[49] Y. Zhang and X.-J. Yang, "An efficient analytical method for solving local fractional nonlinear PDEs arising in mathematical physics," Applied Mathematical Modelling, vol. 40, no. 3, pp. 1793-1799, 2016.

[50] S. Abbasbandy, "Numerical solution of non-linear KleinGordon equations by variational iteration method," International Journal for Numerical Methods in Engineering, vol. 70, no. 7, pp. 876-881, 2007.

[51] S. T. Mohyud-Din and A. Yildirim, "Variational iteration method for solving Klein-Gordon equations," Journal of Applied Mathematics, Statistics and Informatics, vol. 6, pp. 99-106, 2010.

[52] J. Saelao and N. Yokchoo, "The solution of Klein-Gordon equation by using modified Adomian decomposition method," Mathematics and Computers in Simulation, vol. 171, pp. 94-102, 2020.

[53] Sirendaoreji, "A new auxiliary equation and exact travelling wave solutions of nonlinear equations," Physics Letters A, vol. 356, no. 2, pp. 124-130, 2006.

[54] J. Singh, D. Kumar, and S. Rathore, "Application of Homotopy perturbation transform method for solving linear and nonlinear Klein-Gordon equations," Journal of Information and Computing Science, vol. 7, pp. 131-139, 2012.

[55] M. Dehghan and A. Shokri, "Numerical solution of the nonlinear Klein-Gordon equation using radial basis functions," Journal of Computational and Applied Mathematics, vol. 230, no. 2, pp. 400-410, 2009.

[56] S. J. Liao, The proposed homotopy analysis technique for the solution of nonlinear problems, Ph.D thesis, Shanghai Jiao Tong University, Shanghai, China, 1992.

[57] A.-M. Wazwaz, "The tanh and the sine-cosine methods for compact and noncompact solutions of the nonlinear kleingordon equation," Applied Mathematics and Computation, vol. 167, no. 2, pp. 1179-1195, 2005.

[58] X. Li and B. Y. Guo, "A legendre pseudo spectral method for solving nonlinear Klein-Gordon equation," Journal of Computational Mathematics, vol. 15, pp. 105-126, 1997.

[59] H. LI, X.-H. Meng, and B. Tian, "Bilinear form and soliton solutions for the coupled nonlinear Klein-Gordon equations," International Journal of Modern Physics B, vol. 26, no. 15, Article ID 1250057, 2012.

[60] A.-M. Wazwaz, "New travelling wave solutions to the Boussinesq and the Klein-Gordon equations," Communications in Nonlinear Science and Numerical Simulation, vol. 13, no. 5, pp. 889-901, 2008.

[61] A. K. Golmankhaneh, A. K. Golmankhaneh, and D. Baleanu, "On nonlinear fractional Klein-Gordon equation," Signal Processing, vol. 91, no. 3, pp. 446-451, 2011.

[62] M. M. Khader, N. H. Swetlam, and A. M. S. Mahdy, "The chebyshev collection method for solving fractional order Klein-Gordon equation," WSEAS Transactions on Mathematics, vol. 13, pp. 31-38, 2014.

[63] I. N. Sneddon, The Use of Integral Transforms, Tata McGraw Hill Edition, New York, NY, USA, 1974.

[64] G. Adomian, "Modification of the decomposition approach to the heat equation," Journal of Mathematical Analysis and Applications, vol. 124, no. 1, pp. 290-291, 1987.

[65] H. E. Gadain, "Application of double laplace decomposition method for solving singular one dimensional system of hyperbolic equations," The Journal of Nonlinear Science and Applications, vol. 10, no. 1, pp. 111-121, 2017. 
[66] D. Huang, G. Zou, and L. W. Zhang, "Numerical approximation of nonlinear Klein-Gordon equation using an element-free approach," Mathematical Problems in Engineering, vol. 2015, 2015 .

[67] A. Ali, H. Susanto, and J. A. D. Wattis, "Breathing modes of long Josephson junctions with phase-shifts," SIAM Journal on Applied Mathematics, vol. 71, no. 1, pp. 242-269, 2011.

[68] Z. Gul and A. Ali, "Localized modes in a variety of driven long Josephson junctions with phase shifts," Nonlinear Dynamics, vol. 94, no. 1, pp. 229-247, 2018.

[69] A. Ali, H. Susanto, and J. A. D. Wattis, "Decay of bound states in a Sine-Gordon equation with double-well potentials," Journal of Mathematical Physics, vol. 56, no. 5, Article ID 051502, 2015.

[70] Z. Gul, A. Ali, and A. Ullah, "Localized modes in parametrically driven long Josephson junctions with a double-well potential," Journal of Physics A: Mathematical and Theoretical, vol. 52, no. 1, Article ID 015203, 2018.

[71] Z. Gul, A. Ali, and I. Ahmad, "Dynamics of ac-driven sineGordon equation for long Josephson junctions with fast varying perturbation," Chaos, Solitons \& Fractals, vol. 107, pp. 103-110, 2018.

[72] A. Ali, H. Susanto, and J. A. D. Wattis, "Rapidly oscillating AC-driven long Josephson junctions with phase-shifts," Physica D: Nonlinear Phenomena, vol. 246, no. 1, pp. 15-22, 2013.

[73] W. A. Khan, A. Ali, Z. Gul, S. Ahmad, and A. Ullah, "Localized modes in PT-symmetric Sine-Gordon couplers with phase shift," Chaos, Solitons \& Fractals, vol. 139, Article ID 110290, 2020. 